\title{
Erythrocyte microRNA sequencing reveals differential expression in relapsing-remitting multiple sclerosis
}

\author{
Kira Groen ${ }^{1,2}$, Vicki E. Maltby ${ }^{1,2}$, Rodney A. Lea ${ }^{2,3}$, Katherine A. Sanders ${ }^{4}$, J. Lynn Fink ${ }^{5}$, Rodney J. Scott ${ }^{2,6,7}$,
} Lotti Tajouri ${ }^{8}$ and Jeannette Lechner-Scott ${ }^{1,2,9^{*}}$

\begin{abstract}
Background: There is a paucity of knowledge concerning erythrocytes in the aetiology of Multiple Sclerosis (MS) despite their potential to contribute to disease through impaired antioxidant capacity and altered haemorheological features. Several studies have identified an abundance of erythrocyte miRNAs and variable profiles associated with disease states, such as sickle cell disease and malaria. The aim of this study was to compare the erythrocyte miRNA profile of relapsing-remitting MS (RRMS) patients to healthy sex- and age-matched controls.
\end{abstract}

Methods: Erythrocytes were purified by density-gradient centrifugation and RNA was extracted. Following library preparation, samples were run on a HiSeq4000 Illumina instrument (paired-end 100 bp sequencing). Sequenced erythrocyte miRNA profiles ( 9 patients and 9 controls) were analysed by DESeq2. Differentially expressed miRNAs were validated by RT-qPCR using miR-152-3p as an endogenous control and replicated in a larger cohort (20 patients and 18 controls). After logarithmic transformation, differential expression was determined by two-tailed unpaired t-tests. Logistic regression analysis was carried out and receiver operating characteristic (ROC) curves were generated to determine biomarker potential.

Results: A total of 236 erythrocyte miRNAs were identified. Of twelve differentially expressed miRNAs in RRMS two showed increased expression (adj. $p<0.05$ ). Only modest fold-changes were evident across differentially expressed miRNAs. RT-qPCR confirmed differential expression of miR-30b-5p (0.61 fold, $p<0.05)$ and miR-3200-3p ( 0.36 fold, $p<0.01$ ) in RRMS compared to healthy controls. Relative expression of miR-3200-5p (0.66 fold, NS $p=0.096$ ) also approached significance. MiR-3200-5p was positively correlated with cognition measured by audio-recorded cognitive screen $(r=0.60 ; p<0.01)$. MiR-3200-3p showed greatest biomarker potential as a single miRNA (accuracy $=75.5 \%, p<0.01$, sensitivity $=72.7 \%$, specificity $=84.0 \%$ ). Combining miR-3200-3p, miR-3200-5p, and miR-30b-5p into a composite biomarker increased accuracy to $83.0 \%(p<0.05)$, sensitivity to $77.3 \%$, and specificity to $88.0 \%$.

Conclusions: This is the first study to report differences in erythrocyte miRNAs in RRMS. While the role of miRNAs in erythrocytes remains to be elucidated, differential expression of erythrocyte miRNAs may be exploited as biomarkers and their potential contribution to MS pathology and cognition should be further investigated.

Keywords: Erythrocytes, microRNA, Relapsing-remitting multiple sclerosis, Next-generation sequencing

\footnotetext{
* Correspondence: jeannette.lechner-scott@hnehealth.nsw.gov

${ }^{1} \mathrm{School}$ of Medicine and Public Health, University of Newcastle, Callaghan, NSW 2308, Australia

${ }^{2}$ Centre for Information Based Medicine, Level 3 West, Hunter Medical

Research Institute, 1 Kookaburra Circuit, New Lambton Heights, NSW 2305,

Australia

Full list of author information is available at the end of the article
}

(c) The Author(s). 2018 Open Access This article is distributed under the terms of the Creative Commons Attribution 4.0 International License (http://creativecommons.org/licenses/by/4.0/), which permits unrestricted use, distribution, and reproduction in any medium, provided you give appropriate credit to the original author(s) and the source, provide a link to the Creative Commons license, and indicate if changes were made. The Creative Commons Public Domain Dedication waiver (http://creativecommons.org/publicdomain/zero/1.0/) applies to the data made available in this article, unless otherwise stated. 


\section{Background}

Multiple Sclerosis (MS) is an autoimmune disease of the central nervous system (CNS) marked by lymphocytic infiltration, demyelination, and neurodegeneration. It affects approximately 2.5 million individuals worldwide. MS is a heterogeneous disease, which is divided into three disease courses with relapsing-remitting MS (RRMS) being the most common (around $85 \%$ of the patient population) $[1,2]$. Its aetiology is assumed to be the interaction between environmental risk factors and genetic predisposition [3], however the exact cause and pathophysiology remain unclear.

MS is associated with activated peripheral and CNSresident immune cells [2] and it remains to be determined whether erythrocytes play a role in its pathology. Erythrocytes are anucleate cells responsible primarily for respiratory gas transport $[4,5]$, yet are also thought to play a dynamic role in health and disease [6]. The potential involvement of erythrocytes in MS has been recently reviewed [7]. Briefly, altered haemorheological features of erythrocytes have been documented in MS and may be contributing to blood-brain barrier (BBB) disruption, a hallmark of MS pathology [2]. Furthermore, erythrocytes may contribute to increased levels of oxidative stress in MS through impaired antioxidant enzyme capacity [7]. In addition to MS pathology, disease-modifying therapies (DMTs) also appear to affect erythrocytes. For instance, natalizumab has been shown to result in previously undetected circulating erythrocyte precursors [810]. Mitoxantrone [11], fingolimod [12], and dimethyl fumarate [13] all have the potential to cause eryptosis, erythrocyte-specific apoptosis, and interferon- $\beta$ has been shown to reduce red cell distribution width, possibly altering haemorheological features [14].

More recent studies have focused on the potential use of erythrocytes as MS biomarkers, using exogenous Cpeptide binding to erythrocytes [15]. Biomarkers that can accurately reflect pathological and physiological processes are crucial in diseases as complex and heterogeneous as MS. Such biomarkers are needed for diagnosis and patient stratification, but also monitoring of treatment efficacy and disease progression [16]. Current MS diagnosis and monitoring relies on procedures such as lumbar punctures and magnetic resonance imaging (MRI) of the brain and spinal cord [2]. MicroRNA (miRNA) profiles are gaining increasing interest as MS biomarkers as they can reflect a range of ongoing pathological and physiological processes simultaneously [16, 17]. MiRNAs are small ( $22 \mathrm{bp})$ non-coding RNA molecules that control gene expression at the posttranscriptional level [18]. Studies have shown that in peripheral blood mononuclear cell (PBMC) miRNAs can accurately differentiate between MS patients and healthy controls (HCs) $[19,20]$; however, PBMCs only make up a very small percentage of whole blood elements [4]. The majority of a blood sample, erythrocytes and plasma, is discarded when assessing PBMCs. Additionally, some DMTs, such as fingolimod, are known to decrease circulating PBMC numbers [21]. Consequently, PBMC-derived miRNA profiles may not be the most suitable biomarker to monitor MS. Erythrocytes, which are abundant and can be quickly and cost-effectively purified [4], may lend themselves as a superior option.

Recent expression studies have identified an amplitude of miRNA transcripts in circulating erythrocytes [6, 22, 23]. Erythrocyte miRNA profiles were found to differ from leukocyte and reticulocyte profiles, yet largely reflect the miRNA expression of whole blood [6]. While the exact role of miRNAs in translationally inactive erythrocytes [24] is still unknown, they may be involved in intercellular communication through erythrocytederived extracellular vesicles (EVs) [25], or remnants of a functional erythrocyte precursor transcriptome $[6,24]$. Despite uncertainty regarding the function of erythrocyte miRNAs, they have been found to differ in health and disease [6]. The use of erythrocyte-specific miRNA profiles is advantageous to whole blood miRNAs, as it eliminates variation that may arise from differences in whole blood cell composition. Erythrocytes have an average lifespan of 120 days in healthy individuals [4], but reduced lifespans have been reported in athletes [26] and some disease states [27]. Therefore, erythrocyte miRNAs may prove to present a relatively stable picture of miRNA expression, whereas translationally active cell miRNA profiles tend to only provide a snapshot of current miRNA expression, subjective to day-to-day variation [28]. However, this hypothesis demands further investigation.

Erythrocyte miRNAs may be exploited as biomarkers for MS patient stratification, diagnosis, and monitoring of treatment response and disease progression. Nextgeneration sequencing (NGS) technology has the potential to identify novel MS miRNA signatures in erythrocytes. The aim of this study was to characterise the erythrocyte miRNA profile of RRMS patients and compare it to HCs using NGS technologies.

\section{Methods}

\section{Sample collection}

Ethical approval was obtained from the Bond University Human Research Ethics Committee (RO-1382), the University of Newcastle Ethics Committee (H-505-0607), and the Hunter New England Health Ethics Committee (05/04/13/3.09). All participants gave written, informed consent prior to enrolment. Whole blood was collected into EDTA tubes from an initial cohort of 9 female RRMS patients and 9 female healthy controls (HCs). A further 20 female RRMS and 18 female $\mathrm{HC}$ samples 
were collected as part of the replication cohort. RRMS diagnosis was defined according to the McDonald criteria [29]. Participants who were pregnant, breastfeeding, or suffering from an autoimmune condition other than MS were excluded from the study. To minimise confounders associated with sex, only females were recruited for this pilot study. Additional patient information was obtained through MSBase, an observational database open to neurologists and health care teams [30]. Participant characteristics are summarised in Table 1.

\section{Erythrocyte purification}

Erythrocytes were purified from $10 \mathrm{ml}$ whole blood by density-gradient centrifugation. Lymphoprep (Stem Cell Technologies, Canada) density-gradient media was used according to manufacturer protocol. Plasma, PBMC layer, density-gradient media, and top erythrocyte layer were aspirated. The remaining erythrocyte pellet was washed twice with Hanks-balanced salt solution (HBSS) (GE Healthcare, United Kingdom).

\section{Purity assessment of erythrocytes}

Purity of obtained erythrocytes was assessed by flow cytometry. One $\mu \mathrm{l}$ of erythrocyte pellet was stained with FITC-conjugated CD235a (Clone 2B7; erythrocytes and precursors) (BD Pharmingen, USA) and PE-conjugated CD71 (M-A712; reticulocytes) (BD Pharmingen, USA). All samples met a minimum purity cut-off of $95 \%$.
Samples were analysed on FACS Canto II (BD Biosciences, USA) using FACS Diva Software (BD Biosciences, USA).

\section{RNA extraction}

Total RNA was extracted from $300 \mu \mathrm{l}$ erythrocyte pellets with miRNeasy Kits (Qiagen, USA). Pellets were homogenized by vortexing samples for $1 \mathrm{~min}$. Total RNA was quantified using the broad-range RNA Kit for the Qubit 2.0 (Life Technologies, USA). RNA integrity was determined with the RNA 6000 Nano kit on a 2100 Bioanalyzer (Agilent Technologies, USA).

\section{MiRNA sequencing and analysis}

Library preparation and sequencing were performed by the Diamantina Institute, University of Queensland, Brisbane, Australia. Libraries were prepared with TruSeq Small RNA Library Preparation kits (Illumina, USA). Samples were individually barcoded and then sequenced in two multiplexed pools. Samples were run on the HiSeq4000 platform (Illumina, USA) using paired-end sequencing (read length: $100 \mathrm{bp}$; coverage 1 million reads/sample). Sequencing reads were de-multiplexed using CASAVA 1.8 software package (Illumina, USA) and adapter sequences were trimmed using Trim Galore! (https://www.bioinformatics.babraham.ac.uk/ projects/trim_galore/). Reads were aligned against miRBase 21 [31] using STAR [32] and NGS results were analysed by DESeq2 [33]. Significance was adjusted for false discovery rate (FDR) using the Benjamini-Hochberg procedure.

Table 1 Participant characteristics by cohort (sequencing and replication) and group (control subjects and RRMS patients)

\begin{tabular}{|c|c|c|c|c|c|}
\hline & & \multicolumn{2}{|l|}{ Sequencing Cohort } & \multicolumn{2}{|l|}{ Replication Cohort } \\
\hline & & Control Subjects $(n=9)$ & RRMS Patients $(n=9)$ & Control Subjects $(n=18)$ & RRMS Patients $(n=20)$ \\
\hline \multicolumn{2}{|l|}{ Female } & $100 \%$ & $100 \%$ & $100 \%$ & $100 \%$ \\
\hline \multicolumn{2}{|l|}{ Caucasian } & $100 \%$ & $90 \%$ & $94 \%$ & $100 \%$ \\
\hline \multicolumn{2}{|l|}{ Age (years) } & $34.75( \pm 11.45)$ & $42.44( \pm 9.66)$ & $38.92( \pm 9.89)$ & $38.39( \pm 9.41)$ \\
\hline \multicolumn{2}{|c|}{ Disease Duration (years) } & $\mathrm{N} / \mathrm{A}$ & $15.11( \pm 11.87)$ & N/A & $9.29( \pm .5 .52)$ \\
\hline \multicolumn{2}{|l|}{ EDSS Score } & & $2.00( \pm 1.32)$ & & $2.09( \pm 1.53)$ \\
\hline \multicolumn{2}{|c|}{ Age at Onset (years) } & & $27.94( \pm 8.48)$ & & $28.96( \pm 8.79)$ \\
\hline \multicolumn{2}{|c|}{ Number of Relapses } & & $5.98( \pm 4.91)$ & & $6.35( \pm 3.33)$ \\
\hline \multicolumn{2}{|c|}{ Days since Last Relapse } & & $1083.00( \pm 1271.81)$ & & $985( \pm 969.75)$ \\
\hline \multirow[t]{4}{*}{ Treatment } & Off Treatment & & 2 & & 0 \\
\hline & Dimethyl fumarate & & 2 & & 0 \\
\hline & Fingolimod & & 3 & & 10 \\
\hline & Natalizumab & & 2 & & 10 \\
\hline \multicolumn{2}{|c|}{ Time on Treatment (years) } & & $2.46( \pm 2.60)$ & & $3.57( \pm 2.20)$ \\
\hline \multicolumn{2}{|c|}{ Number of Relapses on Treatment } & & $0.56( \pm 0.73)$ & & $1.50( \pm 2.12)$ \\
\hline \multicolumn{2}{|c|}{ Most recent $A R C S^{a}$} & & $78.33( \pm 26.76)$ & & $81.35( \pm 20.43)$ \\
\hline
\end{tabular}

Except for percentages and absolute numbers, all data is presented as mean ( \pm SD). RRMS relapsing-remitting multiple sclerosis, EDSS extended disability status scale, ARCS audio-recorded cognitive screen, SD standard deviation of the mean. ${ }^{\text {a ARCS }}$ are only reported for patients who completed an ARCS within a year of sample collection (sequencing cohort $n=3$; replication cohort $n=17$ ) 
Reverse transcription quantitative polymerase chain reaction (RT-qPCR) - Validation and replication

Differentially expressed erythrocyte miRNAs flagged by NGS were confirmed in the initial NGS cohort and in a replication cohort of 20 RRMS patients and $18 \mathrm{HCs}$ using TaqMan Advanced miRNA Assays (Assay IDs: hsa-let-7f-5p: 478578, hsa-miR-30b-5p: 478007, hsamiR-32-5p: 478026, hsa-96-5p: 478215, hsa-181a-5p: 477857, hsa-miR-362-5p: 478059, hsa-598-3p: 478172, hsa-652-3p: 478189, hsa-miR-660-5p: 478192, hsa-miR1294: 478693, hsa-miR-3200-3p: 478322, hsa-miR-32005p: 478021; Applied Biosystems, Thermo Fisher Scientific, USA). MiR-152-3p (hsa-miR-152-3p: 477921; Applied Biosystems, Thermo Fisher Scientific, USA) was used as an endogenous normalisation control based on a previous study investigating erythrocyte miRNAs by RTqPCR [6]. The expression of miR-152-3p did not differ between RRMS patients and healthy controls in our sequencing cohort (Additional file 1: Table S1). Additionally, miR-152-3p expression was not altered during different stages of erythroid differentiation [34], it demonstrated the least variation across 40 different human tissue samples (Applied Biosystems, unpublished data, see reference [6]), and showed great stability in a hepatic study [34]. Relative expression was calculated using the $2^{\text {-deltaCT }}$ method. All RT-qPCR experiments were performed on a ViiA 7 (Applied Biosystems, USA) instrument. IBM SPSS Statistics 24 was used for statistical analysis. Since this was a relatively small discovery-based project we chose to set a relaxed significance threshold of 0.05 so as to reduce true positive rejection rate (Type II error). Replication of significant hits using an independent replication cohort was performed the reduce the false positive rate (Type I error). Following logarithmic transformation, relative expression determined by RT-qPCR was assessed by two-tailed unpaired Student's t-tests or ANOVA, depending on the number of groups to be compared. Receiver operating characteristic (ROC) curves were generated to assess the diagnostic value of confirmed miRNAs and accuracy was determined by area under the curve (AUC). Using relative expression cut-off values that resulted in greatest sensitivity and specificity for confirmed miRNAs, logistic regression analysis was performed in IBM SPSS Statistics 24 to determine the value of composite biomarkers using multiple confirmed miRNAs. Pearson correlation coefficients between confirmed miRNA and recorded disease outcome measures (Table 1) were calculated.

\section{MiRNA target prediction}

MiRSystem was used to predict target genes of differentially expressed miRNAs as it integrates seven prediction algorithms and includes experimental validation of miRNA-mRNA interactions [35].

\section{Results}

NGS was used to determine erythrocyte miRNA profiles of 9 RRMS patients and 9 HCs. RT-qPCR was then utilised to confirm differential expression of erythrocyte miRNAs revealed by NGS in the sequencing cohort and a more uniform replication cohort of 20 RRMS patients and $18 \mathrm{HC}$ samples. Participant characteristics of the sequencing and replication cohort are shown in Table 1.

\section{Total RNA}

Total erythrocyte RNA was extracted from $300 \mu$ l erythrocyte pellets (mean purity determined by flow cytometry was $99 \%$, with < 1\% leukocytes and platelets; further detail in Additional file 2: Table S2). Total erythrocyte RNA content in $10 \mathrm{ml}$ whole blood was calculated by multiplying RNA yields from one aliquot by the number of aliquots obtained. Mean erythrocyte RNA yield obtained from $10 \mathrm{ml}$ whole blood of MS patients $(n=29)$ was $20.12 \mu \mathrm{g}$ $( \pm 17.02 \mu \mathrm{g})$, while the mean erythrocyte RNA content of HCs $(n=27)$ was $12.44 \mu \mathrm{g}( \pm 3.85 \mu \mathrm{g}) / 10 \mathrm{ml}$ whole blood $(p<0.05)$. RNA yield did not correlated with bench time (time that passed between sample collection and processing) (MS patients: $r=0.145, p=0.553$; HC: $r=0.169, p=0$. 502). Patients treated with natalizumab $(32.35 \mu \mathrm{g} / 10 \mathrm{ml}$ whole blood) had significantly greater RNA yields than patients on fingolimod $(15.52 \mu \mathrm{g} / 10 \mathrm{ml}$ whole blood, $p<0$. $01)$ and HCs $(12.44 \mu \mathrm{g} / 10 \mathrm{ml}$ whole blood, $p<0.001)$ (Additional file 3: Figure S1).

\section{Next-generation sequencing results}

Following TruSeq Small RNA library preparation (Illumina, USA), erythrocyte samples were sequenced by paired-end 100 bp sequencing on a HiSeq4000 (Illumina, USA) instrument, aiming for 1 million reads per sample. NGS revealed 236 known miRNAs across patient and control erythrocytes. Normalised gene counts ranged from 1 to $>21,800$ (Additional file 1: Table S1). Twelve erythrocyte miRNAs were found to be differentially expressed in RRMS patients, two of which, miR-1294 (1.55-fold, FDR adj. $p<0.05)$ and let-7f-5p (1.73-fold, adj. p < 0.05), showed increased expression in RRMS compared to HCs. The remaining ten miRNAs, miR-181a-5p (0.45 fold, adj. $p<0.0001)$, miR-96-5p (0.43 fold, adj. $p<0.05)$, miR-32-5p (0.49 fold, adj. $p<0.05)$, miR-598-3p (0.53 fold, adj. p < 0.05), miR-362-5p (0.59 fold, adj. $p<0.05)$, miR-30b-5p (0.60 fold, adj. $\mathrm{p}<0.05)$, miR660-5p (0.64 fold, adj. $p<0.05)$, miR-652-3p (0.69 fold, adj. $\mathrm{p}<0.05)$, and miR-3200-3p and $-5 \mathrm{p}$ (0.85 fold, adj. $p<0.01)$ , showed decreased expression in RRMS compared to HCs (Fig. 1). Four of the differentially expressed miRNAs (miR181a-5p, miR-362-5p, miR-598-3p, and miR-96-5p) were very lowly expressed ( $<100$ reads) and only let-7f-5p, miR$660-5 p$, and miR-652-3p reached more than 800 reads per sample (Additional file 1: Table S1). No obvious clustering 


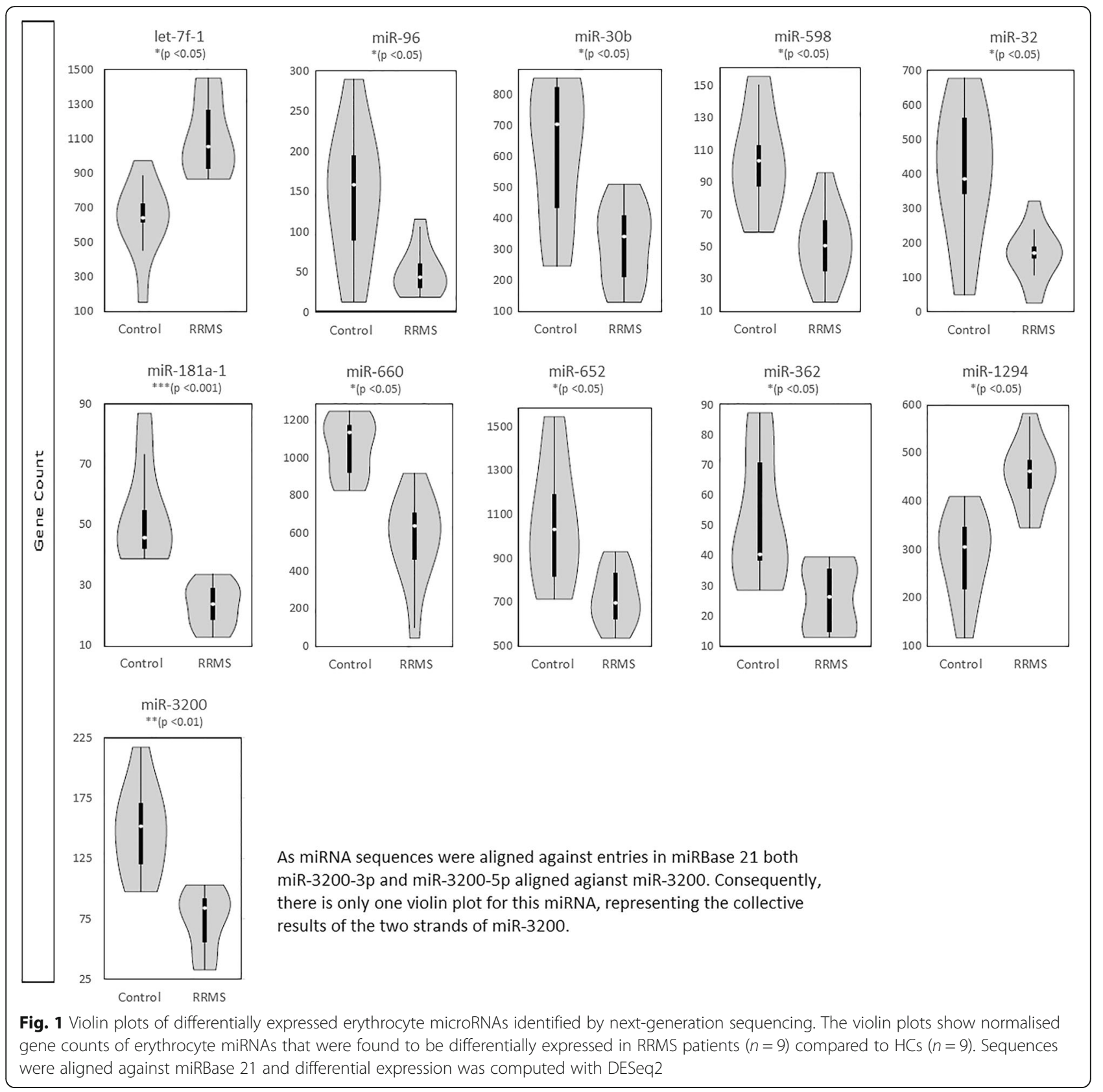

of patients and controls was evident when looking at global differences in miRNA expression.

\section{Reverse transcription quantitative polymerase chain reaction results - Validation and replication}

To validate NGS results, differentially expressed erythrocyte miRNAs were assessed by RT-qPCR in the original sequencing cohort and a replication cohort.

Samples that did not meet our quality control cut-off were removed from the dataset. Differential expression trends in the discovery cohort (9 RRMS patients and 5 $\mathrm{HCs}$ ) were replicated for all miRNAs with the exception of let-7f-5p (Fig. 2). While the significance threshold $(p<0.05)$ was not reached, this may be a result of insufficient power to detect a significant change. With the aim of assessing whether differential erythrocyte miRNA expression was driven by DMTs as opposed to disease, the original sequencing cohort was segregated by DMT. No formal analysis was carried out due to lack of power, yet visual representation shows that with the exception of miR-3200-3p, miR3200-5p, and miR-652-3p, off treatment RRMS patients' erythrocyte miRNA expression resembles that of patients on DMT (Additional file 4: Figure S2). 


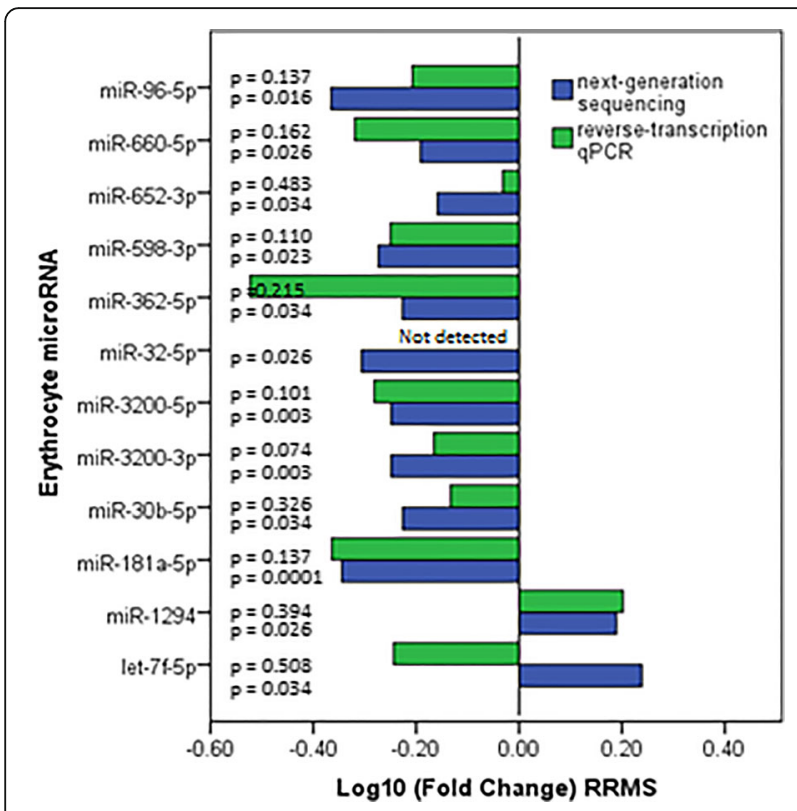

Fig. 2 Log10(fold changes) of differentially expressed erythrocyte microRNAs in relapsing-remitting Multiple Sclerosis. Log10(fold changes) of differentially expressed erythrocyte miRNAs in relapsing-remitting Multiple Sclerosis (RRMS) patients compared to healthy controls (HC) by next-generation sequencing (9 RRMS patients and $9 \mathrm{HC}$ ) (blue) and RT-qPCR (9 RRMS patients and $5 \mathrm{HC}$ ) (green)

RT-qPCR experiments were replicated in a larger, more uniform cohort of 20 RRMS patients and 18 HCs. Decreased expression of miR-30b-5p $(0.61$ fold, $p<0.05)$ and miR-3200-3p ( 0.36 fold, $p<0.01)$ was confirmed. Decreased expression of miR-3200-5p (0.66 fold, NS $p=0.096$ ) approached significant threshold and was hence included in further analysis (Fig. 3). To assess the biomarker potential of the confirmed miRNAs, ROC curve analysis was performed for miRNAs that showed differential expression. ROC curves graphically illustrate the diagnostic potential of a binary outcome, in this case RRMS or $\mathrm{HC}$, at different thresholds. This allows the determination of true and false positives for each of the thresholds, and specificity and sensitivity to be calculated.

Cut-off values that maximised both specificity and sensitivity were chosen. MiR-3200-3p showed the greatest biomarker potential being able to distinguish between RRMS patients and HCs with $75.5 \%$ accuracy $(p<0.01$; relative expression cut-off: 0.81 ), $72.7 \%$ sensitivity, and $84.0 \%$ specificity. MiR-30b-5p and miR-3200-5p were able to differentiate between RRMS and HCs with 70.5\% $(\mathrm{p}<0.05)$ and $65.8 \%(p=0.064)$ accuracy, $68.2 \%$ and $59.1 \%$ sensitivity, and $72.0 \%$ and $68.0 \%$ specificity respectively (Fig. 4; Table 2). Combining relative expression (using cut-off values in Table 2) of the three confirmed miRNAs (miR30b-5p, miR-3200-3p, and miR-3200-5p) in a binary logistic regression with $\mathrm{MS}$ or $\mathrm{HC}$ being the dichotomous

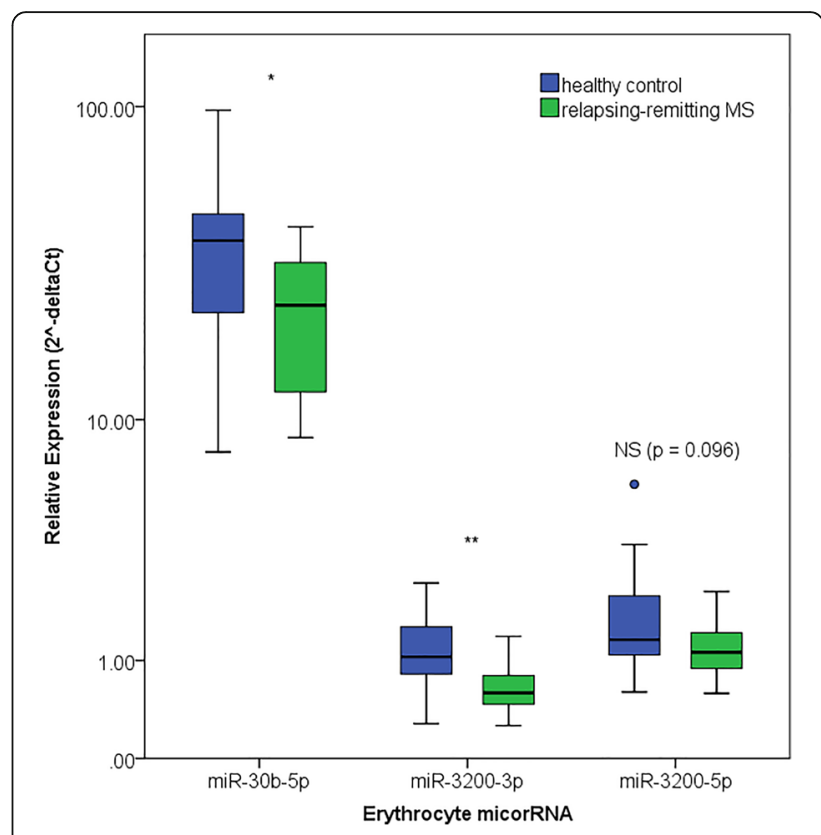

Fig. 3 Tukey boxplot of differentially expressed erythrocyte microRNAs confirmed by reverse transcription polymerase chain reaction. Tukey boxplot of relative expression ( $2^{\text {-deltacT }}$ ) ( $y$-axis on a log scale) of erythrocyte microRNAs in relapsing-remitting MS patients $(n=20$; green) and healthy controls ( $n=18$; blue). The blue dot represents an outlier defined as deviating $\geq 1.5$ fold from the upper/lower quartile. ${ }^{*} p<0.05$; ** $p<0.01$; NS - not significant $(p>0.05)$

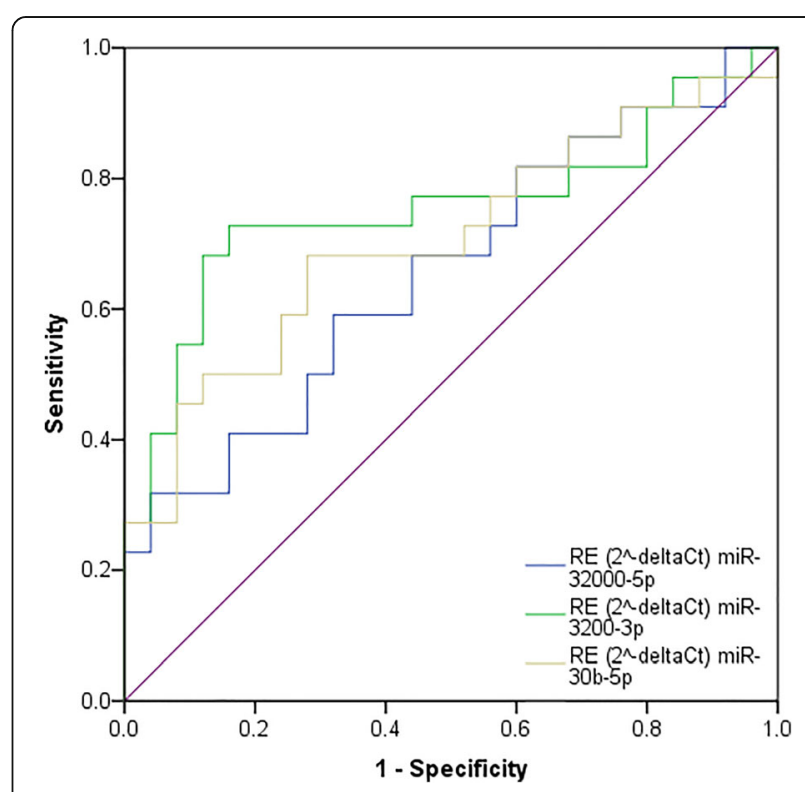

Fig. 4 Receiver operating characteristic (ROC) curves for confirmed erythrocyte microRNAs. ROC curves showing sensitivity and 1-specificity at different thresholds for miR-3200-3p (green), miR-3200-5p (blue), and miR-30b-5p (yellow) 
Table 2 Receiver operating characteristic curve (ROC) results for the three confirmed erythrocyte microRNAs

\begin{tabular}{llllll}
\hline MicroRNA & RE Cut-Off & Sensitivity (\%) & Specificity (\%) & Accuracy (\%) & $p$-value \\
\hline miR-30b-5p & 34.40 & 68.2 & 72.0 & 70.5 & 0.016 \\
miR-3200-3p & 0.81 & 72.7 & 84.0 & 75.5 & 0.003 \\
miR-3200-5p & 1.20 & 59.1 & 68.0 & 65.8 & 0.064 \\
\hline
\end{tabular}

$R E$ relative expression ( $\left.2^{\text {-deltact }}\right)$

outcome, cases and controls could be assigned to their respective category with overall accuracy of $83.0 \%, 77.3 \%$ sensitivity, and $88.0 \%$ specificity (Table 3 ).

To determined clinical impact of differentially expressed erythrocyte miRNAs Pearson correlation coefficients between confirmed miRNAs and recorded disease outcome measures (Table 1) were calculated. Relative expression of miR-3200-5p was positively correlated with patients most recent (within a year of sample collection) cognitive assessment measured by audiorecorded cognitive screen (ARCS) [36] (Pearson correlation coefficient: 0.597; $\mathrm{p}<0.01$ ) (Fig. 5), as well as some of the ARCS' subdomains (Table 4). No further correlations between confirmed miRNAs and disease outcome measures, age, or bench time (elapsed time between blood collection and processing) were identified.

There were no significant differences in miRNA levels between patients on natalizumab $(n=12)$ and fingolimod $(n=13)$ across the 12 assessed erythrocyte miRNAs.

\section{MiRNA target prediction}

Given that fold changes were only modest for all differentially expressed miRNAs (Fig. 2), it is unlikely that a single miRNA is significantly affecting target gene expression. To this end, it was reasoned that several miRNAs may work in concert to target a few specific messenger RNAs (mRNAs). MiRSystem [35] was used to identify genes targeted by several of the 12 differentially expressed miRNAs identified by NGS. MiRSystem identified several genes targeted by four of the miRNAs flagged by NGS and one gene, MIER 3 (mesoderm induction early response 1 , family member 3 ), was targeted by five of the miRNAs identified as differentially expressed by NGS (Table 5).

Table 3 Classification of relapsing-remitting Multiple Sclerosis patients and healthy controls based on three erythrocyte miRNAs

\begin{tabular}{lllll}
\hline & & Predicted & & $\begin{array}{l}\text { Percentage } \\
\text { Correct (\%) }\end{array}$ \\
\cline { 3 - 4 } & RRMS & HC & \\
\hline Observed & RRMS & 22 & 3 & 88.0 \\
& HC & 5 & 17 & 77.3 \\
Overall percentage & & & 83.0
\end{tabular}

RRMS relapsing-remitting Multiple Sclerosis, $H C$ healthy control

\section{Discussion}

This is the first study to compare the erythrocyte miRNA profile of RRMS patients to HCs. Differentially expressed erythrocyte miRNAs were identified in both the sequencing and replication cohort. Three miRNAs (miR-30b-5p, miR-3200-3p and miR-3200-5p) were found to show decreased expression in RRMS erythrocytes compared to $\mathrm{HC}$ erythrocytes and the combination of these miRNAs (miR-30b-5p, miR-3200-3p and miR3200-5p) into a composite biomarker, was able to differentiate between RRMS patients and healthy controls with $77.3 \%$ sensitivity, $88.0 \%$ specificity, overall accuracy of $83.0 \%$. Additionally, miR-3200-5p showed moderate correlation with patients' cognitive function, determined by ARCS (correlation coefficient: $0.60 ; p<0.01$ ).

Current MS diagnosis is based on the 2013 revisions to the McDonald criteria and clinical evidence is a crucial component of this diagnosis [29]. Diagnosis and monitoring of MS is underpinned by demyelinating lesions on MRI and supported by positive oligoclonal bands in patients' cerebrospinal fluid (CSF), requiring an invasive lumbar puncture. There is no reliable blood test that may guide diagnosis, monitoring, and selection of treatment options $[1,2]$. Differential expression of miRNAs in erythrocytes, which can be easily obtained as

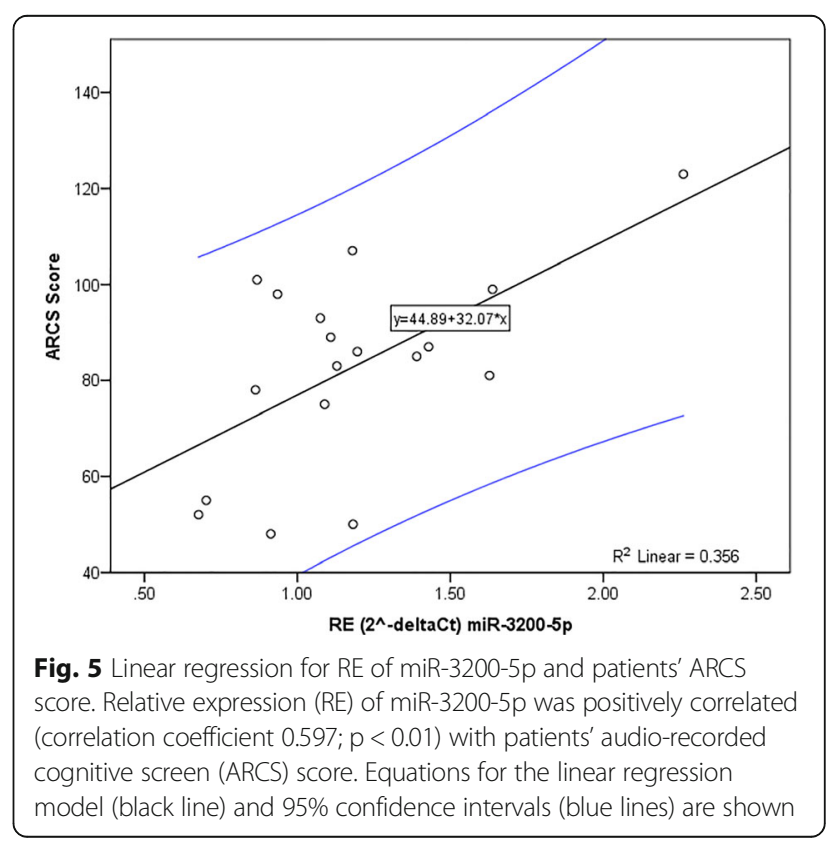


Table 4 Pearson correlation coefficients between miR-3200-5p and ARCS scores and subdomains

\begin{tabular}{lll}
\hline & Pearson Correlation Coefficient & $p$-value \\
\hline Total ARCS & $\mathbf{0 . 5 9 7}$ & $\mathbf{0 . 0 0 9}$ \\
Quick ARCS & $\mathbf{0 . 5 9 2}$ & $\mathbf{0 . 0 1 0}$ \\
Memory Domain & 0.427 & 0.077 \\
Fluency Domain & $\mathbf{0 . 4 9 9}$ & $\mathbf{0 . 0 3 5}$ \\
Visuospatial Domain & 0.314 & 0.205 \\
Language Domain & 0.282 & 0.256 \\
Attention Domain & 0.391 & 0.108 \\
Speed of Writing & $\mathbf{0 . 6 3 4}$ & $\mathbf{0 . 0 0 5}$ \\
\hline
\end{tabular}

ARCS audio-recorded cognitive screen; significant correlations are highlighted in bold

part of other routine blood tests, indicates potential as supportive biomarkers for MS diagnosis and monitoring, reducing cost and patient discomfort associated with current paraclinical investigations. Erythrocytes are abundant in whole blood and thought to be translationally inactive; therefore, they lend themselves as stable biomarkers and should be further investigated. In order to establish the true diagnostic potential of erythrocyte miRNAs, their expression in MS needs to be compared to other differential diagnoses, this should be addressed by future studies. Differential diagnoses for MS include vascular diseases, such as systemic vasculitis, conditions of the brain and spinal cord, such as cerebellar ataxias, and tumours and structural lesions in the CNS [3]. Thus far, erythrocyte miRNAs have not been studied in diseases that make up the differential diagnoses for MS. Nonetheless, a study investigating whole blood miRNA profiles in CIS/RRMS patients and patients with neuromyelitis optica spectrum disorders (NMOSD) found differential expression of miR-30b in their discovery cohort (20 CIS/RRMS patients and 20 NMOSD patients) and replication cohort (19 RRMS/CIS patients and 18 NMOSD patients) [37]. As miR-30b was also found to be differentially expressed in this study and whole blood
miRNAs have been found to reflect erythrocyte miRNAs [6], the aforementioned finding [37] underpins the potential erythrocyte miRNAs have as diagnostic biomarkers for MS. Studies have also compared whole blood miRNA profiles between stroke patients and healthy controls, highlighting differential expression patterns [38] and focusing on let-7e-5p expression [39]. With the differential expression patterns between stroke patients and healthy controls [38, 39] differing from the differential erythrocyte miRNA expression between healthy controls and RRMS patients identified by this study, one may argue that miRNAs can differentiate between stroke and RRMS patients, however this needs to be confirmed through further investigation.

NGS of erythrocyte miRNAs of female RRMS patients and $\mathrm{HCs}$ revealed 236 known miRNAs across RRMS and $\mathrm{HC}$ samples. Several of the miRNAs flagged by NGS, including the confirmed miR-3200-3p, have also been identified in Alzheimer's Disease (AD). Satoh et al. identified decreased expression of let-7f-5p, miR-660-5p, miR-1294, and miR-3200-3p, as well as several others [40]. While not all trends match those in RRMS (Fig. 2), involvement of the same miRNAs in two distinct neurological diseases suggests their overall importance for the CNS. Satoh et al. used datasets from whole blood samples [40], not isolated erythrocytes, which raises the possibility of lymphocyte contamination. However, a previous study has shown that whole blood miRNAs largely reflect erythrocyte miRNAs [6], allowing for comparison between the two studies. Thus far, isolated erythrocyte miRNAs have not been studied in diseases other than malaria [41] and sickle cell disease [6].

One of the major symptoms of MS is cognitive impairment, which may develop in the absence of clinical relapse [2, 3]. Appropriate management of cognitive impairment is only possible if it can be detected early in the affected population [42]. Conventional neuropsychological assessment, the gold standard for detecting changes in cognition, is time-consuming, requires a

Table 5 MicroRNA target prediction results

\begin{tabular}{lll}
\hline Target Gene & Gene Description & Observed microRNA \\
\hline MIER 3 & mesoderm induction early response 1, family member 3 & miR-181a-5p, miR-30b-5p, miR-32-5p, miR-362-5p, miR-660-5p \\
BCL2L11 & BCL2-like 11 (apoptosis facilitator) & miR-181a-5p, miR-30b-5p, miR-32-5p, miR-362-5p \\
SEC24A & SEC24, family member A & miR-181a-5p, miR-30b-5p, miR-32-5p, miR-660-5p \\
SCN3A & sodium channel, voltage-gated type III, alpha subunit & miR-30b-5p, miR-32-5p, miR-362-5p, miR-660-5p \\
BCL11A & B-cell CLL/lymphoma 11A (zinc finger protein) & miR-181a-5p, miR-30b-5p, miR-32-5p, miR-362-5p \\
YTHDF3 & YTH domain family, member 3 & miR-181a-5p, miR-30b-5p, miR-362-5p, miR-660-5p \\
NFAT5 & nuclear factor of activated T-cells 5, tonicity response & miR-181a-5p, miR-30b-5p, miR-32-5p, miR-660-5p \\
CPEB4 & cytoplasmic polyadenylation element binding protein 4 & miR-181a-5p, miR-30b-5p, miR-32-5p, miR-660-5p \\
LIN28 & & miR-181a-5p, miR-30b-5p, miR-32-5p, miR-598-3p \\
CNTN4 & contactin 4 & miR-181a-5p, miR-30b-5p, miR-32-5p, miR-362-5p
\end{tabular}


trained psychologist, and is not feasible for routine clinical practice. Consequently, other cognitive screening instruments, such as the ARCS, have been developed; nonetheless, the ARCS still requires patients to spend $35 \mathrm{~min}$ in a quiet room [36]. No blood-borne biomarker for cognitive impairment has been implemented in clinical practice [43]. Differential expression of miR-3200-5p was correlated with patients' most recent ARCS score (correlation coefficient: 0.597, $p<0.01$ ), indicating that this miRNA may serve as a biomarker for cognitive function, reflecting global neuronal loss. The strongest correlation was observed between the subdomain speed of writing and the miRNA (correlation coefficient: 0.634, $\mathrm{p}<0.01$ ). Speed of writing requires patients to write out the word "table" as many times as possible in $30 \mathrm{~s}$, reflecting information processing and fine motor skills [36]. The idea that circulating miRNAs may reflect cognitive impairment is not new: two small studies looked at mild cognitive impairment in the elderly and identified some miRNAs that correlated with mild cognitive impairment with high sensitivity and specificity $[42,43]$. Cognitive decline is an early sign of MS and may reflect CNS damage more accurately than EDSS scores $[44,45]$. Early detection for timely treatment and management are key to improve outcomes in MS, yet current cognitive screens are time and labour intensive and can be distressing for patients. The correlation between miR3200-5p and patients' ARCS score indicates this miRNA's potential to be developed into a biomarker for cognitive impairment. Longitudinal assessment and validation in a larger cohort are necessary to confirm this hypothesis.

While a range of targets were predicted by miRSystem [35], fold changes in differentially expressed miRNAs were only minor, reducing the likelihood that a single miRNA is affecting gene expression. Nonetheless, several of the differentially expressed miRNAs were found to target the same genes, potentially amplifying dysregulation [18]. None of the predicted targets play an established role in mature erythrocytes and MIER3 is not known to play a role in MS. The role of miRNAs in translationally inactive mature erythrocytes remains to be elucidated [24]. It has been suggested that erythrocyte miRNAs are remnants from earlier stages of erythrocyte development, where they played crucial roles in cell differentiation and maturation [6, 22]. Notwithstanding, erythrocyte miRNAs may play a more active role, functioning as intercellular communicators through erythrocyte-derived EVs. EVs are small, membranebound vesicles, containing proteins, nucleic acids, and lipids, which can be derived from a variety of cells, including erythrocytes [25].

None of the differentially expressed erythrocyte miRNAs were found to be highly abundant in erythrocytes (some of the most abundant miRNAs were miR-25,
miR-144, miR-451, miR-182, and members of the let-7 family; Additional file 1: Table S1) and the reason for the observed differential expression remains to be clarified. Potential angles for investigation include stabilisation of certain miRNAs through associations with protein complexes, such as Argonaute proteins [22] and other noncoding RNAs [18], as well as targeted packaging and loss of certain miRNAs through EVs [25].

Recruited patients were on various DMTs (Table 1), some of which are known to alter erythrocyte phenotypes [8-13]. To account for the treatment effects, patients were recruited on a range of therapies, with the intent of identifying miRNA signatures that were disease- rather than treatment-specific. While lack of power did not allow for formal comparisons between RRMS patients on DMTs and untreated RRMS patients, visual comparison indicated that expression of miR-3200-3p, miR-3200-5p, and miR-652-3p might differ between these groups (Additional file 4: Figure S2). These differences and the effect of DMTs on erythrocyte miRNA expression needs to be further investigated and some of the findings of this study may be specific to MS patients on DMTs. Differences between DMTs were also assessed. Differential expression between natalizumab and fingolimod treated patients was evident for let-7f-5p, which showed increased expression in patients on fingolimod compared to patients on natalizumab (data not shown). While not statistically significant, this difference may explain why the trend of differential expression could not be replicated by RT-qPCR for let-7f-5p (Fig. 2). Neither of the treatment groups showed differential let-7f-5p expression compared to healthy controls (data not shown). Members of the let-7 family have been reported in other erythrocyte miRNA studies [6, 22, 23], where they are thought to be involved in erythropoiesis [46], and have also been shown to be less expressed in MS patients [47]. Cox et al. [47] used PAXGene (Qiagen, Germany) technology to analyse whole blood miRNA expression. Lack of similarity between identified miRNAs, other than let-7f, by Cox et al. and in this study, may reflect differences in cell make up, with PAXGene technology focussing mostly on leukocytes. Nevertheless, the reason for the difference in miRNA expression between treatments remains unknown and warrants further investigation.

Total RNA obtained from $10 \mathrm{ml}$ whole blood varied between 9.40 and $35.35 \mu \mathrm{g}$ (Additional file 1: Figure S1). Both reticulocytes and nucleated cells have been shown to harbour greater amounts of total RNA than erythrocytes [6]. Increased RNA yields from erythrocytes of patients treated with natalizumab may reflect increased levels of circulating erythrocyte precursors in these patients [8-10].

While low power to detect small differences in miRNA species and the recruitment of patients on different DMTs, some of which are known to alter erythrocytes [8-13], should be addressed by future studies, these 
preliminary results indicate that erythrocyte miRNAs should be incorporated into the growing list of MS biomarkers. Future investigations should aim to recruit larger numbers of treatment-naïve patients (including male MS patients), assess intra-individual variability, and evaluate specificity of observed differential expression to MS. In addition to healthy controls, future studies should aim to recruit pathological controls suffering from other systemic inflammatory, neurodegenerative, and autoimmune diseases.

The potential importance of erythrocyte pellets to MS and other diseases is starting to be recognized: Erythrocyte/granulocyte pellets are already being stored as part of the UK ME/CFS Biobank [48] and future investigations into erythrocytes and MS, or other autoimmune diseases, may provide a novel avenue for immunoregulatory prophylaxis and treatment options [49].

\section{Conclusions}

This is the first study to explore erythrocyte miRNAs in RRMS. We find evidence to suggest that erythrocyte miRNAs, particularly miR-30b-5p, miR-3200-3p and miR-3200-5p, may be exploited as novel MS biomarkers and miR-3200-5p may be developed into a biomarker for cognitive decline. Further investigations are warranted to substantiate these findings as erythrocytes can be easily and cost-effectively purified and novel biomarkers are required to aid diagnosis and stratification of MS patients.

\section{Additional files}

Additional file 1: Table S1. Next-generation sequencing results of erythrocyte microRNAs for 9 healthy controls and 9 relapsing-remitting Multiple Sclerosis patients. (XLSX $27 \mathrm{~kb}$ )

Additional file 2: Table S2. Erythrocyte purity determined by flow cytometry $(n=10)$. Data is shown as mean percent positive events out of 20,000 events. (XLSX $12 \mathrm{~kb}$ )

Additional file 3: Figure S1. Total erythrocyte RNA extracted from $10 \mathrm{ml}$ whole blood by disease-modifying therapy. Mean total erythrocyte RNA yields from $10 \mathrm{ml}$ whole blood for 2 patients off treatment, 2 on dimethyl fumarate, 13 on fingolimod, 12 on natalizumab and 27 healthy controls. Error bars represent standard deviation (SD). ${ }^{* *} p<0.01 ;{ }^{* * *} p<0.001$. (PNG $41 \mathrm{~kb}$ )

Additional file 4 Figure $\mathbf{S 2}$. Tukey boxplot of relative expression (2-deltact) of differentially expressed erythrocyte microRNAs in the sequencing cohort by disease-modifying therapy. Relative expression ( $\left.2^{\text {-deltact }}\right)(y$-axis on a logarithmic scale) of differentially expressed erythrocyte miRNAs ( $x$-axis) by disease-modifying therapy (fingolimod: $n=3$; natalizumab: $n=2$; dimethyl fumarate: $n=2$; off treatment: $n=2)$ and including healthy controls $(n=5)$. The dots represent outliers defined as deviating $\geq 1.5$ fold from the upper/ lower quartile. (PNG $29 \mathrm{~kb}$ )

\section{Abbreviations}

AD: Alzheimer's disease; ARCS: Audio-recorded cognitive screen; AUC: Area under the curve; BBB: Blood-brain barrier; CNS: Central nervous system; CSF: Cerebrospinal fluid; DMT: Disease-modifying therapy; EDSS: Extended disability status scale; EV: Extracellular vesicle; FDR: False discovery rate; HBSS: Hanks' balanced salt solution; HC: Healthy control; miRNA: microRNA;
MRI: Magnetic resonance imaging; mRNA: messenger RNA; MS: Multiple Sclerosis; NGS: Next-generation sequencing; NMOSD: Neuromyelitis optica spectrum disorder; PBMC: Peripheral blood mononuclear cell; RE: Relative expression; ROC: Receiver operating characteristic; RRMS: Relapsing-remitting Multiple Sclerosis; RT-qPCR: Reverse transcription quantitative polymerase chain reaction; SD: Standard deviation of the mean

\section{Acknowledgements}

The authors would like to acknowledge PhD candidate Sharon Song and Dr. Lisa Anderson for performing the NGS experiments. We would like to acknowledge Dr. Karen Ribbons for her assistance in establishing the miRNA-ARCS correlation. Further, we would like to thank Susan Agland and Trish Collinson for assisting with sample collection and laboratory work. We would like to extend our gratitude to all MS patients and control participants that volunteered to be part of this study.

\section{Funding}

This project was funded by Bond University and an MSRA (MS Research Australia) Grant. K Groen is funded by a scholarship from the University of Newcastle and was funded by a partial scholarship from Bond University at the time of sample collection. KA Sanders was funded by a scholarship from Multiple Sclerosis Research Australia and the Trish Multiple Sclerosis Research Foundation. VE Maltby is funded by fellowships from Multiple Sclerosis Research Australia and the Canadian Institutes of Health Research.

\section{Availability of data and materials}

The dataset supporting the conclusions of this article will be released upon request.

\section{Authors' contributions}

KG and LT, who had the original idea, drafted the project proposal. KG drafted the manuscript, processed the majority of the samples, carried out the method optimisation and RT-qPCR experiments, put together figures and tables and performed statistical analysis. VEM edited the manuscript, processed some of the samples and supervised KG in the laboratory. RAL and LF carried out analysis of the NGS data and provided guidance for further statistical analysis. KAS aided sample collection and processing. All authors participated in the editing and drafting of the manuscript. JLS recruited patients through the John Hunter hospital and supervised the project with RJS. All authors read and approved the final manuscript.

\section{Ethics approval and consent to participate}

Ethical approval was obtained from the Bond University Human Research Ethics Committee (RO-1382), the University of Newcastle Ethics Committee (H-505-0607), and the Hunter Area Research Ethics Committee (no. 05/04/13/ 3.09). All participants gave written informed consent prior to enrolment.

\section{Competing interests}

JLS's institution receives non-directed funding, as well as honoraria for presentations and membership on advisory boards from Sanofi Aventis, Biogen Idec, Bayer Health Care, Merck Serono, Teva, Roche, and Novartis Australia.

\section{Publisher's Note}

Springer Nature remains neutral with regard to jurisdictional claims in published maps and institutional affiliations.

\section{Author details}

'School of Medicine and Public Health, University of Newcastle, Callaghan, NSW 2308, Australia. ${ }^{2}$ Centre for Information Based Medicine, Level 3 West, Hunter Medical Research Institute, 1 Kookaburra Circuit, New Lambton Heights, NSW 2305, Australia. ${ }^{3}$ Institute of Health and Biomedical Innovations, Genomics Research Centre, Queensland University of Technology, Kelvin Grove, QLD 4059, Australia. ${ }^{4}$ Centre for Anatomical and Human Sciences, Hull York Medical School, Hull HU6 7RX, UK. ${ }^{5}$ Diamantina Institute, University of Queensland, Woolloongabba, QLD 4102, Australia. ${ }^{6}$ Division of Molecular Genetics, Pathology North, John Hunter Hospital, New Lambton Heights, NSW 2305, Australia. ${ }^{7}$ School of Biomedical Sciences and Pharmacy, University of Newcastle, Callaghan, NSW 2308, Australia. ${ }^{8}$ Faculty of Health Sciences and Medicine, Bond University, QLD, Robina 4229, Australia. 
${ }^{9}$ Department of Neurology, John Hunter Hospital, New Lambton Heights, NSW 2305, Australia.

\section{Received: 24 January 2018 Accepted: 1 May 2018 Published online: 21 May 2018}

\section{References}

1. Lublin FD, Reingold SC, Cohen JA, Cutter GR, Sorensen PS, Thompson AJ, Wolinsky JS, Balcer LJ, Banwell B, Barkhof F, et al. Defining the clinical course of multiple sclerosis: the 2013 revisions. Neurology. 2014;83(3):278-86.

2. Dendrou CA, Fugger L, Friese MA. Immunopathology of multiple sclerosis. Nat Rev Immunol. 2015;15(9):545-58.

3. Compston A Coles A. Multiple sclerosis. Lancet. 2008:372(9648):1502-17.

4. Marieb EN, Hoehn K. Human anatomy \& physiology. Boston: Pearson; 2013.

5. Klinken SP. Red blood cells. Int J Biochem Cell Bio. 2002:34(12):1513-8.

6. Chen SY, Wang Y, Telen MJ, Chi JT. The genomic analysis of erythrocyte microRNA expression in sickle cell diseases. PlosOne. 2008;3(6):e2360.

7. Groen K, Maltby VE, Sanders KA, Scott RJ, Tajouri L, Lechner-Scott J. Erythrocytes in multiple sclerosis-forgotten contributors to the pathophysiology? Mult Scler J Exp Transl Clin. 2016;2:2055217316649981.

8. Bridel C, Beauverd Y, Samii K, Lalive PH. Hematologic modifications in natalizumab-treated multiple sclerosis patients: an 18-month longitudinal study. Neurol Neuroimmunol Neuroinflamm. 2015;2(4):123.

9. Jing D, Oelschlaegel U, Ordemann R, Holig K, Ehninger G, Reichmann H, Ziemssen T, Bornhauser M. CD49d blockade by natalizumab in patients with multiple sclerosis affects steady-state hematopoiesis and mobilizes progenitors with a distinct phenotype and function. Bone Marrow Transplant. 2010;45(10):1489-96.

10. Lesesve JF, Debouverie M, Decarvalho Bittencourt M, Bene MC. CD49d blockade by natalizumab therapy in patients with multiple sclerosis increases immature B-lymphocytes. Bone Marrow Transplant. 2011:46(11):1489-91.

11. Arnold M, Bissinger R, Lang F. Mitoxantrone-induced suicidal erythrocyte death. Cell Physiol Biochem. 2014;34(5):1756-67.

12. Eberhard M, Ferlinz K, Alizzi K, Cacciato PM, Faggio C, Foller M, Lang F. FTY720-induced suicidal erythrocyte death. Cell Physiol Biochem. 2010; 26(4-5):761-6

13. Ghashghaeinia M, Bobbala D, Wieder T, Koka S, Bruck J, Fehrenbacher B, Rocken M, Schaller M, Lang F, Ghoreschi K. Targeting glutathione by dimethylfumarate protects against experimental malaria by enhancing erythrocyte cell membrane scrambling. Am J Physiol Cell Physiol. 2010; 299(4):791-804.

14. Peng YF, Cao WY, Zhang Q, Chen D, Zhang ZX. Assessment of the relationship between red cell distribution width and multiple sclerosis. Medicine (Baltimore). 2015:94(29):e1182.

15. Lockwood SY, Summers S, Eggenberger E, Spence DM. An in vitro diagnostic for multiple sclerosis based on C-peptide binding to erythrocytes. EBioMedicine. 2016;11:249-52.

16. Bielekova B, Martin R. Development of biomarkers in multiple sclerosis. Brain. 2004;127(7):1463-78.

17. Raphael I, Webb J, Stuve O, Haskins WE, Forsthuber TG. Body fluid biomarkers in multiple sclerosis: how far we have come and how they could affect the clinic now and in the future. Expert Rev Clin Immunol. 2015;11(1):69-91.

18. Esteller M. Non-coding RNAs in human disease. Nat Rev Genet. 2011;12(12): 861-74

19. Keller A, Leidinger P, Lange J, Borries A, Schroers H, Scheffler M, Lenhof HP, Ruprecht K, Meese E. Multiple sclerosis: microRNA expression profiles accurately differentiate patients with relapsing-remitting disease from healthy controls. PlosOne. 2009:4(10):e7440.

20. Otaegui D, Baranzini SE, Armananzas R, Calvo B, Munoz-Culla M, Khankhanian P, Inza I, Lozano JA, Castillo-Trivino T, Asensio A, et al. Differential micro RNA expression in PBMC from multiple sclerosis patients. PlosOne. 2009;4(7):e6309.

21. Chun J, Hartung HP. Mechanism of action of oral fingolimod (FTY720) in multiple sclerosis. Clin Neuropharmacol. 2010;33(2):91-101.

22. Azzouzi I, Moest H, Wollscheid B, Schmugge M, Eekels JJ, Speer O. Deep sequencing and proteomic analysis of the microRNA-induced silencing complex in human red blood cells. Exp Hematol. 2015:43(5):382-92.

23. Doss JF, Corcoran DL, Jima DD, Telen MJ, Dave SS, Chi JT. A comprehensive joint analysis of the long and short RNA transcriptomes of human erythrocytes. BMC Genomics. 2015;16(1):952.
24. Goh SH, Lee YT, Bouffard GG, Miller JL. Hembase: browser and genome portal for hematology and erythroid biology. Nucleic Acids Res. 2004; 32(suppl 1):572-4

25. Zaborowski MP, Balaj L, Breakefield XO, Lai CP. Extracellular vesicles: composition, biological relevance, and methods of study. Bioscience. 2015; 65(8):783-97.

26. Smith J. Exercise, training and red blood cell turnover. Sports Med. 1995; 19(1):9-31.

27. Lang E, Lang F. Triggers, inhibitors, mechanisms, and significance of eryptosis: the suicidal erythrocyte death. Biomed Res Int. 2015;2015:513518.

28. Daniels SI, Sillé FCM, Goldbaum A, Yee B, Key EF, Zhang L, Smith MT, Thomas R. Improving power to detect changes in blood miRNA expression by accounting for sources of variability in experimental designs. Cancer Epidemiol Biomark Prev. 2014;23(12):2658-66.

29. Polman CH, Reingold SC, Banwell B, Clanet M, Cohen JA, Filippi M, Fujihara K, Havrdova E, Hutchinson M, Kappos L, et al. Diagnostic criteria for multiple sclerosis: 2010 revisions to the McDonald criteria. Ann Neurol. 2011:69(2): 292-302.

30. Butzkueven H, Chapman J, Cristiano E, Grand'Maison F, Hoffmann M, Izquierdo G, Jolley D, Kappos L, Leist T, Pöhlau D, et al. MSBase: an international, online registry and platform for collaborative outcomes research in multiple sclerosis. Mult Scler J. 2006;12(6):769-74.

31. Kozomara A, Griffiths-Jones S. miRBase: annotating high confidence microRNAs using deep sequencing data. Nucleic Acids Res. 2014; 42(Database issue):D68-73.

32. Dobin A, Davis CA, Schlesinger F, Drenkow J, Zaleski C, Jha S, Batut P, Chaisson $M$, Gingeras TR. STAR: ultrafast universal RNA-seq aligner. Bioinformatics. 2013:29(1):15-21.

33. Love MI, Huber W, Anders S. Moderated estimation of fold change and dispersion for RNA-seq data with DESeq2. Genome Biol. 2014;15(12):550

34. Choong ML, Yang HH, McNiece I. MicroRNA expression profiling during human cord blood-derived CD34 cell erythropoiesis. Exp Hematol. 2007; 35(4):551-64.

35. Lu T-P, Lee C-Y, Tsai M-H, Chiu Y-C, Hsiao CK, Lai L-C, Chuang EY. miRSystem: an integrated system for characterizing enriched functions and pathways of MicroRNA targets. PlosOne. 2012;7(8):e42390.

36. Schofield PW, Lee SJ, Lewin TJ, Lyall G, Moyle J, Attia J, McEvoy M. The audio recorded cognitive screen (ARCS): a flexible hybrid cognitive test instrument. J Neurol Neurosurg Psychiatry. 2010;81(6):602-7.

37. Keller $A$, Leidinger $P$, Meese $E$, Haas J, Backes $C$, Rasche L, Behrens JR, Pfuhl C, Wakonig K, Giess RM, et al. Next-generation sequencing identifies altered whole blood microRNAs in neuromyelitis optica spectrum disorder which may permit discrimination from multiple sclerosis. J Neuroinflammation. 2015;12:196.

38. Tan KS, Armugam A, Sepramaniam S, Lim KY, Setyowati KD, Wang CW, Jeyaseelan K. Expression profile of MicroRNAs in young stroke patients. PlosOne. 2009:4(11):e7689.

39. Huang S, Lv Z, Guo Y, Li L, Zhang Y, Zhou L, Yang B, Wu S, Zhang Y, Xie C, et al. Identification of Blood Let-7e-5p as a Biomarker for Ischemic Stroke. PlosOne. 2016;11(10):e0163951.

40. Satoh J, Kino Y, Niida S. MicroRNA-Seq data analysis pipeline to identify blood biomarkers for Alzheimer's disease from public data. Biomark Insights. 2015;10:21-31

41. Mantel PY, Hjelmqvist D, Walch M, Kharoubi-Hess S, Nilsson S, Ravel D, Ribeiro M, Gruring C, Ma S, Padmanabhan P, et al. Infected erythrocytederived extracellular vesicles alter vascular function via regulatory Ago2miRNA complexes in malaria. Nat Commun. 2016;7:12727.

42. Kayano M, Higaki S, Satoh Jl, Matsumoto K, Matsubara E, Takikawa O, Niida S. Plasma microRNA biomarker detection for mild cognitive impairment using differential correlation analysis. Biomarker Res. 2016;4:22.

43. Sheinerman KS, Tsivinsky VG, Abdullah L, Crawford F, Umansky SR. Plasma microRNA biomarkers for detection of mild cognitive impairment: biomarker validation study. Aging. 2013;5(12):925-38

44. Feuillet L, Reuter F, Audoin B, Malikova I, Barrau K, Cherif AA, Pelletier J. Early cognitive impairment in patients with clinically isolated syndrome suggestive of multiple sclerosis. Mult Scler. 2007:13(1):124-7.

45. DeLuca GC, Yates RL, Beale H, Morrow SA. Cognitive impairment in multiple sclerosis: clinical, radiologic and pathologic insights. Brain Pathol. 2015;25(1):79-98.

46. Lawrie $\mathrm{CH}$. microRNA expression in erythropoiesis and erythroid disorders. Br J Haematol. 2010;150(2):144-51. 
47. Cox MB, Cairns MJ, Gandhi KS, Carroll AP, Moscovis S, Stewart GJ, Broadley S, Scott RJ, Booth DR, Lechner-Scott J. MicroRNAs miR-17 and miR-20a inhibit T cell activation genes and are under-expressed in MS whole blood. PlosOne. 2010:5(8):e12132.

48. Lacerda EM, Bowman EW, Cliff JM, Kingdon CC, King EC, Lee JS, Clark TG, Dockrell HM, Riley EM, Curran H, et al. The UK ME/CFS biobank for biomedical research on Myalgic encephalomyelitis/chronic fatigue syndrome (ME/CFS) and multiple sclerosis. Open J Bioresour. 2017:4.

49. Pishesha N, Bilate AM, Wibowo MC, Huang NJ, Li Z, Dhesycka R, Bousbaine D, Li H, Patterson HC, Dougan SK, et al. Engineered erythrocytes covalently linked to antigenic peptides can protect against autoimmune disease. Proc Natl Acad Sci U S A. 2017;114(12):3157-62.

Ready to submit your research? Choose BMC and benefit from:

- fast, convenient online submission

- thorough peer review by experienced researchers in your field

- rapid publication on acceptance

- support for research data, including large and complex data types

- gold Open Access which fosters wider collaboration and increased citations

- maximum visibility for your research: over $100 \mathrm{M}$ website views per year 